\title{
A novel risk score model based on eight genes and a nomogram for predicting overall survival of patients with osteosarcoma
}

\author{
Guangzhi Wu' and Minglei Zhang ${ }^{2^{*}}$
}

\begin{abstract}
Background: This study aims to identify a predictive model to predict survival outcomes of osteosarcoma (OS) patients.

Methods: A RNA sequencing dataset (the training set) and a microarray dataset (the validation set) were obtained from The Cancer Genome Atlas (TCGA) and Gene Expression Omnibus (GEO) database, respectively. Differentially expressed genes (DEGs) between metastatic and non-metastatic OS samples were identified in training set. Prognosis-related DEGs were screened and optimized by support vector machine (SVM) recursive feature elimination. A SVM classifier was built to classify metastatic and non-metastatic OS samples. Independent prognosic genes were extracted by multivariate regression analysis to build a risk score model followed by performance evaluation in two datasets by Kaplan-Meier (KM) analysis. Independent clinical prognostic indicators were identified followed by nomogram analysis. Finally, functional analyses of survival-related genes were conducted.

Result: Totally, 345 DEGs and 45 prognosis-related genes were screened. A SVM classifier could distinguish metastatic and non-metastatic OS samples. An eight-gene signature was an independent prognostic marker and used for constructing a risk score model. The risk score model could separate OS samples into high and low risk groups in two datasets (training set: log-rank $p<0.01, \mathrm{C}$-index $=0.805$; validation set: $\log$-rank $p<0.01, \mathrm{C}$-index $=$ 0.797). Tumor metastasis and RS model status were independent prognostic factors and nomogram model exhibited accurate survival prediction for OS. Additionally, functional analyses of survival-related genes indicated they were closely associated with immune responses and cytokine-cytokine receptor interaction pathway.
\end{abstract}

Conclusion: An eight-gene predictive model and nomogram were developed to predict OS prognosis.

Keywords: Osteosarcoma, Differentially expressed genes, Support vector machine, Risk score, Prognosis

\footnotetext{
*Correspondence: zhangml1997@126.com

2Departments of Orthopedics, The Third Hospital of Jilin University, Changchun, Jilin Province, China

Full list of author information is available at the end of the article
}

(C) The Author(s). 2020 Open Access This article is licensed under a Creative Commons Attribution 4.0 International License, which permits use, sharing, adaptation, distribution and reproduction in any medium or format, as long as you give appropriate credit to the original author(s) and the source, provide a link to the Creative Commons licence, and indicate if changes were made. The images or other third party material in this article are included in the article's Creative Commons. licence, unless indicated otherwise in a credit line to the material. If material is not included in the article's Creative Commons licence and your intended use is not permitted by statutory regulation or exceeds the permitted use, you will need to obtain permission directly from the copyright holder. To view a copy of this licence, visit http://creativecommons.org/licenses/by/4.0/ The Creative Commons Public Domain Dedication waiver (http://creativecommons.org/publicdomain/zero/1.0/) applies to the data made available in this article, unless otherwise stated in a credit line to the data. 


\section{Background}

Osteosarcoma (OS) is a common malignant bone cancer in adolescents around the whole world and has a high tendency of metastasis [1]. The considerable progress in OS prevention and treatment has been made by introducing promising therapeutic strategies such as postoperative neo-adjuvant chemotherapy and multi-agent systemic chemotherapy over the past decades $[2,3]$. However, the statistical evidence suggested that the incidence and mortality rates of OS have been continuously growing by approximately $1.4 \%$ each year [4]. The recent studies demonstrated that the 5-year survival rate of OS remains about $65 \%$ and more than half of OS patients die from OS metastasis [5, 6]. Therefore, the identification of novel prognostic gene markers for metastasis of OS is imperative for improving the overall survival of OS patients.

The advent of next sequencing technologies allows rapid disease detection and diagnosis in recent decades. Accordingly, extensive studies based on the microarray data and transcriptome sequencing data were carried out to identify potential gene drivers involved in the occurrence, metastasis and recurrence of tumors. Notably, existing evidence has showed that numerous gene signatures had significant prognostic values for OS. Wang et al argued that OS patients with a high $A L D H 1 B 1$ level had an unfavorable clinical outcome compared to those OS patients with low $A L D H 1 B 1$ level, implying that this gene might be a potential prognostic marker for patients with OS [7]. Shi et al examined the expression difference and prognostic power of $D D X 10$ based on a dataset from Gene Expression Omnibus (GEO) database. They found that there was a higher expression level of DDX10 in OS tissues than normal tissues and increased $D D X 10$ level was related to a poor prognosis [8]. Furthermore, a previous research analyzed three miRNA expression profiles and constructed a support vetor mechine (SVM) classifier with 15 differentially expressed miRNAs (DEmiRNAs). The results showed that this classifier had a relatively high accuracy to predict OS recurrence, suggesting that these DEmiRNAs were possibly associated with OS prognosis [9]. Liu et al recently screened a fourpseudogene signature for OS survival prediction based on the RNA sequencing data and this pseudogene panel could clearly differentiate high and low risk patients with OS [10]. Although previous studies have identified many gene makers in the development and recurrence, a deeper understanding of the influence of gene signatures on the survival prognosis of OS needs further investigating.

In the present research, the differentially expressed genes (DEGs) between metastatic and non-metastatic OS samples were identified from the training dataset obtained from The Cancer Genome Atlas (TCGA) database. Then, the prognostic genes were screened followed by optimized selection based on SVM recursive feature elimination
(SVM-RFE) algorithm. The optimal prognostic genes were used to construct a SVM classifier to separate OS metastatic and non-metastatic OS samples. Additionally, a machine learning analysis (univariate and multivariate cox regression) was used to extract independent OS prognostic genes to construct a risk score (RS) model. The independent clinical prognostic indicator was identified followed by a predictive nomogram construction. Finally, the functional analyses of prognosis-related genes were also performed. Our findings will promote the understanding of clinical prognostic outcomes of OS patients.

\section{Methods}

\section{Data source}

The level 3 mRNA sequencing data of OS patients was firstly downloaded from TCGA (https://gdc-portal.nci.nih. gov/) database, which was generated by Illumina HiSeq 2000 RNA sequencing platform and concluded 265 samples. Subsequently, we mapped these samples into their clinical characteristics obtained and removed those samples without metastasis information. Finally, a total of 176 OS samples (58 metastatic samples and 118 nonmetastatic samples) were included in this study. Accordingly, the mRNA sequencing data from these samples and corresponding clinical information were retained and considered as the training dataset in following analysis. In addition, the OS gene expression matrix from GSE21257 dataset provided by Buddingh et al [11] was acquired from GEO [12] repository (http://www.ncbi.nlm.nih.gov/geo/). This dataset was based on Illumina human-6 v2.0 expression beadchip platform and comprised of 53 OS samples (34 samples with OS metastasis and 19 samples without OS metastasis). Moreover, these samples all had the relevant clinical prognosis information, and therefore, we regarded this dataset as the validation dataset.

\section{Identification of differentially expressed genes (DEGs)}

For the training dataset, all samples were divided into two groups (metastatic and non-metastatic group). Then, we employed the limma [13] package (version 3.34.7; https:// bioconductor.org/packages/release/bioc/html/limma.html) in $\mathrm{R}$ 3.4.1 to identify the significantly DEGs between OS metastasis samples and OS without metastasis samples according to the thresholds of false discovery rate (FDR) < 0.05 and the $\mid \log _{2}$ fold change (FC) $\mid>0.5$. Furthermore, the bidirectional hierarchical clustering analysis of DEGs in training dataset was carried out based on the centered pearson correlation algorithm using the pheatmap [14] package (version 1.0.8; https://cran.r-project.org/web/ packages/pheatmap/index.html) in $\mathrm{R}$ 3.4.1.

\section{Screening of prognostic genes for OS}

Firstly, the univariate cox regression analysis was performed to extract key genes associated with overall 
survival using the survival [15] package (version 2.41.1; http://bioconductor.org/packages/survivalr/) in $\mathrm{R}$ 3.4.1. The $\log$-rank $p$ value $<0.05$ was thought of as the cutoff for the significant correlation. Afterwards, the e1071 [16] package (version 1.7.1; https://cran.r-project.org/web/ packages/e1071) and caret [17] package (version 6.0.76; https://cran.r-project.org/web/packages/caret) in R 3.4.1 were utilized to further screen the optimal prognostic gene set (OPGS) for OS on the basis of SVM-RFE method, which is an iterative backward selection algorithm and can recursively remove one feature gene with the smallest ranking score until the optimal feature gene set was remained [18]. Following this, the SVM classifier was constructed for predicting OS metastasis based on the expression levels of OPGS. Moreover, the external GSE21257 dataset was used to verify the results of SVM classification analysis. The partial receiver operating characteristic (pROC) [19] package (version 1.15.0; https://cran.r-project.org/web/packages/pROC/index.

html) was utilized to conduct performance evaluation of SVM classifier in training and validation dataset in $R$ 3.4.1. Accordingly, the multiple quantization parameters in classification assessment task were computed, including sensitivity, specificity, area under curve (AUC), positive predictive value (PPV) and negative predictive value (NPV).

\section{Construction of prognostic model for OS and performance evaluation}

The multivariate cox regression analysis was carried out to extract independent prognostic genes for OS using survival package in R 3.4.1 according to the cutoff criterion of the log-rank $p$ value $<0.05$. Afterwards, a risk score model of the prognostic mRNA makers was established according to following formula: Risk score (RS) = $\Sigma \beta_{\text {DEGs }} \times \operatorname{Exp}_{\text {DEGs. }}$ The $\beta_{\text {DEGs }}$ represented the estimated contribution coefficient of independent prognostic mRNAs in the multivariate Cox regression analysis and Exp $_{D E G s}$ denoted the level of independent prognostic genes. According to this formula, the RS of each OS patient was computed. Then, all patients in training dataset were divided into high and low risk groups with the median of the RS as the cut-off criterion. Furthermore, the survival differences between these two risk groups were evaluated by a Kaplan-Meier (KM) survival curve using the survival package in R 3.4.1. Meanwhile, the expression values of independent prognostic genes were also extracted from validation set and the RS for each sample was then calculated. Accordingly, the samples in validation dataset were also divided into two groups (high-risk and low-risk groups) based on the same strategy above mentioned. Finally, the difference in survival prognosis between the high-and low-risk group was also assessed according to three indicators (Harrell C-index, Brier score and $\log$-rank $p$ value of cox proportional hazards regression) [20-22]. Specifically, Harrell C-index, and Brier score were computed by the survcomp [23] package (version 1.30.0; http://www.bioconductor.org/packages/release/bioc/html/survcomp.html) in R 3.4.1. The R survival package was used to undertake the KM estimates of survival probability and calculate the corresponding log-rank $p$ values [15].

\section{Screening of independent prognostic factors}

The univariate and multivariate cox regression analyses were employed to identify the independent clinical prognostic factors using the survival package in $\mathrm{R}$ 3.4.1 with $\log$-rank $p$ value $<0.05$ as the threshold for significance [15]. Following this, we further explored the relationships between independent prognostic factors and survival prognosis. A nomogram was constructed based on independent prognosis-related genes and prognostic factors to predict survival rates of patients at 3 and 5 years via the rms package (version 5.1.2; https://cran.r-project. $\mathrm{org} /$ web/packages/rms/index.html) in R 3.4.1 [22, 24].

\section{Functional analyses of key genes in high and low risk groups}

The samples from training dataset were divided into high-risk and low-risk groups according to the RS in prognostic model. We then used $\mathrm{R}$ limma package to identify the DEGs between these two groups according to $\mathrm{FDR}<0.05$ and $\left|\log _{2} \mathrm{FC}\right|>0.5$ [13]. Subsequently, the Gene Ontology (GO)-biological process (BP) analysis and the Kyoto Encyclopedia of Genes and Genomes (KEGG) pathway enrichment analysis of these genes were carried out using the clusterProfiler [25] package (version 3.6.0; http://bioconductor.org/packages/release/ bioc/html/clusterProfiler.html) in $\mathrm{R}$ 3.4.1. The FDR $<$ 0.05 was considered as the threshold for significant enrichment.

\section{Results \\ DEGs identification}

This study was carried out as showed in Fig. 1. In total, 345 DEGs were identified between metastatic and nonmetastatic OS samples in training group, which contained 48 up-regulated genes and 297 down-regulated genes (Table S1). The volcano plot of these DEGs was displayed in Fig. 2a. In addition, the bidirectional hierarchical clustering analysis was carried out based on the expression profiles of these DEGs. All samples were divided into two groups (metastasis and non-metastasis). We found that 58 metastatic OS samples were all grouped into metastatic cluster. Meanwhile, most of non-metastatic OS samples (111/118) were clustered and seven non-metastatic OS samples were misclassified into metastatic cluster. The accuracy of metastasis 


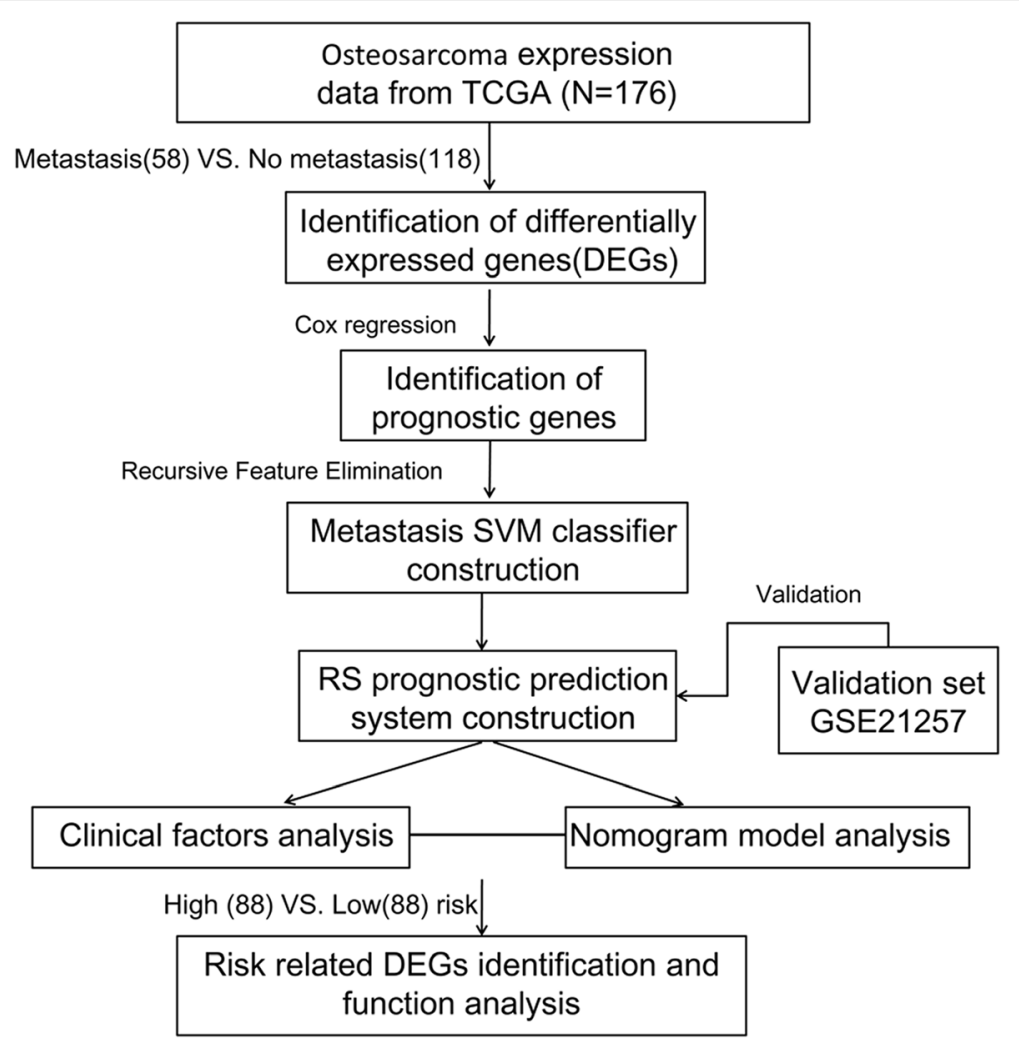

Fig. 1 The flow chart of the whole analysis in this study

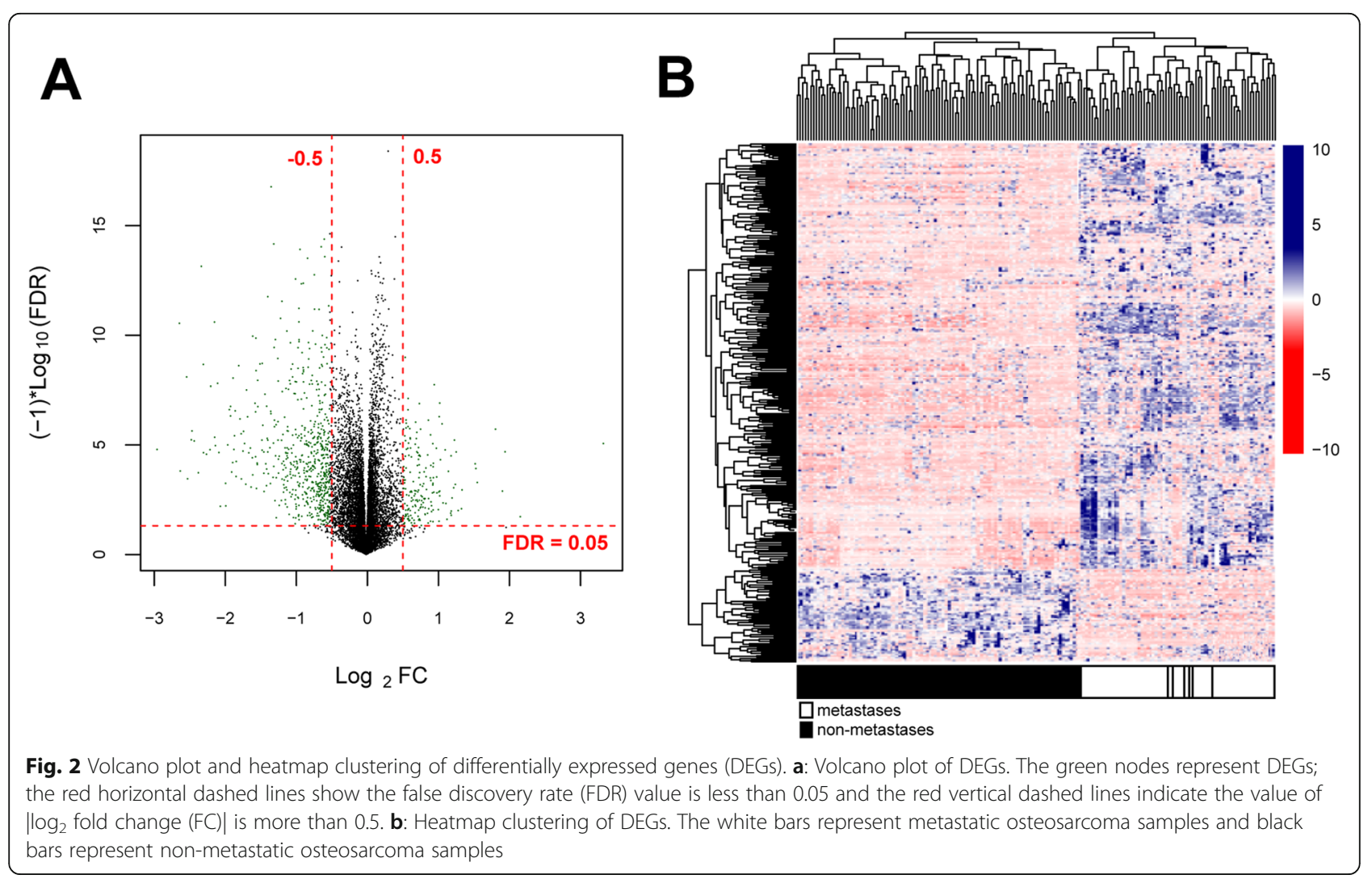


identification was $96 \%$, suggesting that these DEGs exhibited a good discriminative ability for differentiating the metastatic and non-metastatic OS samples (Fig. 2b).

\section{Screening of prognostic feature genes for OS}

The univariate cox regression analysis was performed to identify prognostic genes, and the results revealed that 56 DEGs were significantly correlated with overall survival of patients with OS. To obtain the most representative prognostic genes for metastatic OS, the SVM-RFE method was used to extract the OPGS. Consequently, a total of 45 DEGs were representatively prognosis-related genes with maximum accuracy of 0.901 as showed in Fig. 3a. Furthermore, a SVM-based classifier was constructed on the basis of these 45 DEGs according to the strategy described in method. The performance assessment analysis of the SVM classifier indicated that it could effectively distinguish metastatic OS from non-metastatic OS samples in training dataset with multiple evaluation indicators (the AUC of 0.969 , the sensitivity of 0.915 , the specificity of 0.884 , the PPV of 0.741 and the NPV of 0.966; Table 1; Fig. 3a and b). Similarly, this classifier also exhibited a good discrimination between metastatic and non-metastatic OS samples in validation dataset based on the relatively high values of evaluation index (the AUC of 0.907 , the sensitivity of 0.857 , the specificity of 0.778 , the PPV of 0.882 and the NPV of 0.737 ; Table 1; Fig. 3a and b). These results suggested that this OPGS was predominately associated with the survival outcomes for OS patients and the SVM classifier based on OPGS had a clinical implication for OS metastasis diagnosis.

\section{Screening of independent prognostic feature DEGs and risk model construction}

The multivariate cox regression analysis was conducted to obtain the independent prognostic feature genes. As
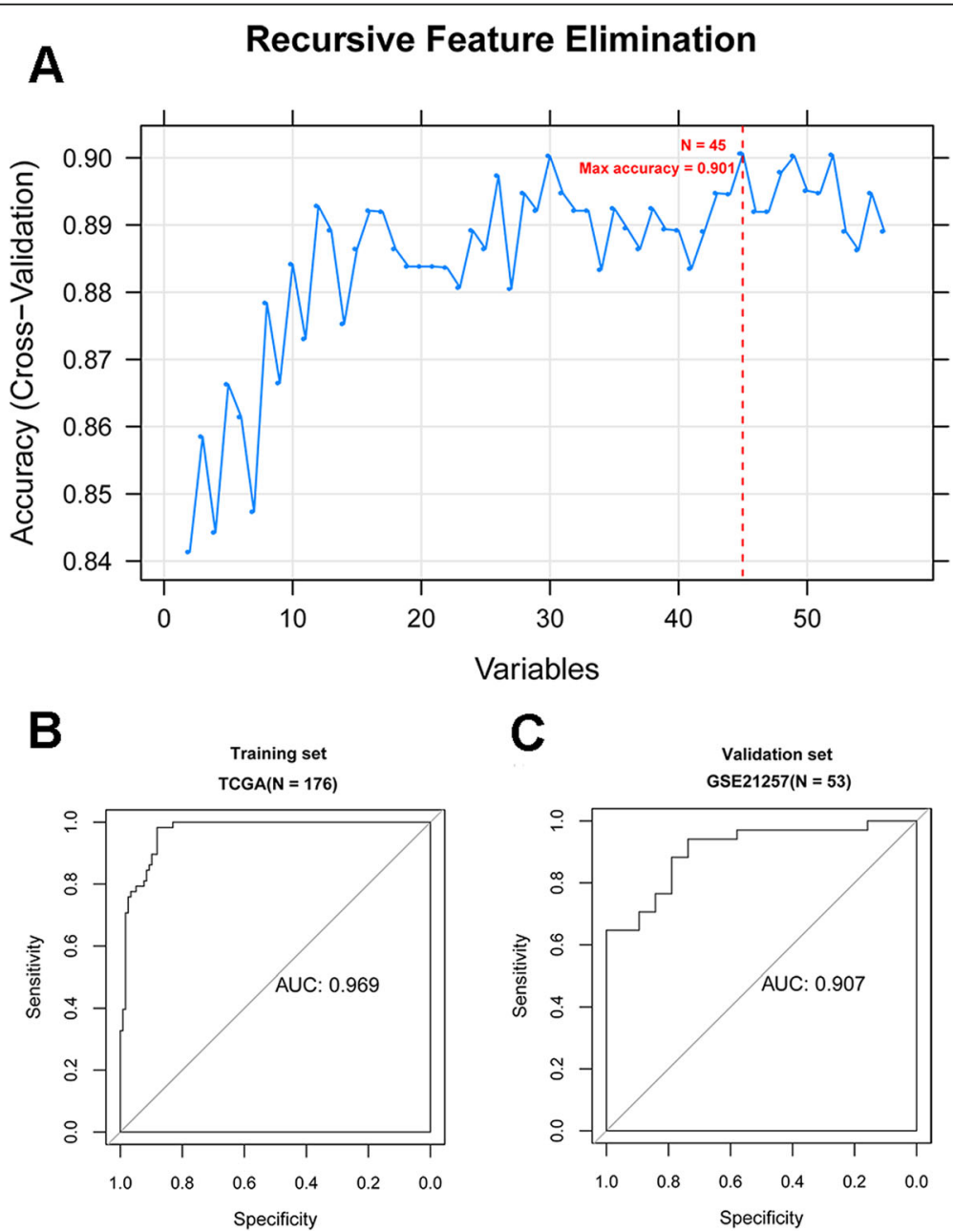

Fig. 3 Screening of the optimal prognostic gene set for osteosarcoma and the receiver operating characteristic (ROC) curve of SVM classification. a: The identification of optimal prognostic gene set for osteosarcoma based on the recursive feature elimination algorithm. The horizontal axis shows the number of differentially expressed genes and the vertical axis represents the cross-validation accuracy. $\mathbf{b}$ : The ROC curve of SVM classification in training dataset. c: The ROC curve of SVM classification in validation dataset 
Table 1 The performance evaluation of a SVM classifier in training and validation dataset

\begin{tabular}{|c|c|c|c|c|c|}
\hline \multirow[b]{2}{*}{ Datasets } & \multicolumn{5}{|l|}{$\mathrm{ROC}$} \\
\hline & AUROC & Sensitivity & Specificity & PPV & NPV \\
\hline Training set $(T C G A, N=176)$ & 0.969 & 0.915 & 0.884 & 0.741 & 0.966 \\
\hline Validation set (GSE21257, $N=53$ ) & 0.907 & 0.857 & 0.778 & 0.882 & 0.737 \\
\hline
\end{tabular}

shown in Table 2, eight genes were found to be independently related to OS prognosis, including $\mathrm{KCNJ15}$ (potassium voltage-gated channel subfamily $\mathrm{J}$ member 15), SLC24A4 (solute carrier family 24 member 4), ASPA (aspartoacylase), REM1 (RRAD and GEM like GTPase 1), SCARA5 (scavenger receptor class A member 5), LANCL3 (LanC like 3), CPA6 (carboxypeptidase A6) and $T R H$ (thyrotropin releasing hormone). Afterwards, the expression levels of these genes in training dataset were computed and RS prediction model was constructed as follows: $\quad R S=(0.0501) \times \operatorname{Exp}_{K C N I 15}+(-0.392) \times$ $\operatorname{Exp}_{\mathrm{SLC24A} 4}+(0.0661) \times \operatorname{Exp}_{\mathrm{ASPA}}+(-0.0633) \times$ $\operatorname{Exp}_{\text {REM } 1}+(-0.024) \times \operatorname{Exp}_{\text {SCARA } 5}+(0.143) \times$ Exp $_{\text {LANCL } 3}+$ $(0.0522) \times \operatorname{Exp}_{\mathrm{CPA} 6}+(0.0592) \times \operatorname{Exp}_{\mathrm{TRH}}$. The RS for each sample was then calculated in training set. All samples $(n=176)$ were classified into high risk group $(n=88)$ and low risk group $(n=88)$ in the light of the median value of RS. The survival analysis revealed that there was a significant correlation between two different risk groups and survival outcomes (Hazard Ratio [HR] = 3.130, 95\% Confidence intervals [CI]: 1.824-5.370, logrank $p=1.275 \mathrm{e}-0.5$, Harrell C-index $=0.805$ and Brier score $=0.049$; Fig. 4a). Meanwhile, the samples $(n=53)$ in validation set were also stratified into high-risk group $(n=27)$ and low-risk group $(n=26)$ using the same method. The dramatic relationship was observed between these risk groups and clinical survival in validation set $(\mathrm{HR}=2.524,95 \% \mathrm{CI}: 1.058-6.020$, log-rank $p=$ 3.101e-02, Harrell C-index $=0.797$ and Brier score $=$ 0.078; Fig. 4b). Notably, we found that the patients from low risk group had higher survival probabilities than

Table 2 The list of independent prognostic feature genes

\begin{tabular}{lllll}
\hline Gene & coef & $\boldsymbol{P}$ value & Hazard Ratio & $95 \% \mathrm{Cl}$ \\
\hline KCNJ15 & 0.0501 & $2.220 \mathrm{E}-03$ & 1.0513 & $1.0182-1.0856$ \\
SLC24A4 & -0.392 & $4.840 \mathrm{E}-03$ & 0.6757 & $0.5145-0.8875$ \\
ASPA & 0.0661 & $7.220 \mathrm{E}-03$ & 1.0683 & $1.0180-1.1211$ \\
REM1 & -0.0633 & $2.152 \mathrm{E}-02$ & 0.9386 & $0.8893-0.9907$ \\
SCARA5 & -0.024 & $2.287 \mathrm{E}-02$ & 0.9763 & $0.9564-0.9967$ \\
LANCL3 & 0.143 & $3.370 \mathrm{E}-02$ & 1.1533 & $1.0111-1.3155$ \\
CPA6 & 0.0522 & $3.787 \mathrm{E}-02$ & 1.0536 & $1.0029-1.1067$ \\
TRH & 0.0592 & $4.298 \mathrm{E}-02$ & 1.0610 & $1.0019-1.1236$ \\
\hline
\end{tabular}

Coef Coefficient derived from multiivariate cox regression analysis, 95\% Cl 95\% confidence interval those patients from high risk group in both training and validation set (Fig. 4).

\section{Predictive nomogram model of independent prognostic factors}

To identify the independent prognostic indicators for OS survival, the univariate and multivariate regression analysis was performed based on the clinical features from of patients in training set. As indicated in Table 3, two independent prognostic parameters (tumor metastasis and RS model status) were remarkably linked with the OS clinical outcomes. Moreover, survival analysis showed that tumor metastasis was markedly related to overall survival (log-rank $p=1.36 \mathrm{e}-05$ and $\mathrm{HR}=2.898$, 95\% CI: 1.754-4.789) and patients with OS metastasis had a worse prognosis than those with OS nonmetastasis, which was in accordance with clinical practice (Fig. 5a). Besides, there were also strong correlations between tumor metastasis and overall survival time in high and low risk groups (for high risk group: log-rank $p=2.455 \mathrm{e}-02$ and $\mathrm{HR}=2.398,95 \% \mathrm{CI}: 1.092-5.268$; for low risk group: log-rank $p=2.189 \mathrm{e}-03$ and $\mathrm{HR}=2.761$, 95\% CI: 1.410-5.405; Fig. 5b and c). Subsequently, the tumor metastasis and RS model status were incorporated into a nomogram model for predicting the rates of overall survival at the 3- and 5-year in OS patients. The score of every indicator can be found by points scale located at the top of nomogram. Then, the points of each indicator were summed, thereby estimating survival probability at 3- and 5-year (Fig. 5d). Furthermore, we constructed a calibration curve to evaluate the performance of nomogram model. Notably, the C-index was respectively 0.695 and 0.683 for OS prediction at the 3and 5-year, suggesting that the nomogram prediction for survival rates was in line with the actual observation for OS patients (Fig. 5e). These results demonstrated that the nomogram based on OS metastasis and RS model status exhibited a good predictive accuracy for survival prognosis of OS patients.

\section{Screening of prognosis risk-related genes and functional analyses}

The samples from training set were separated into high and low risk groups on the basis of RS model. The 614 DEGs were extracted between these two groups using the limma package in R 3.4.1, which comprised of 117 


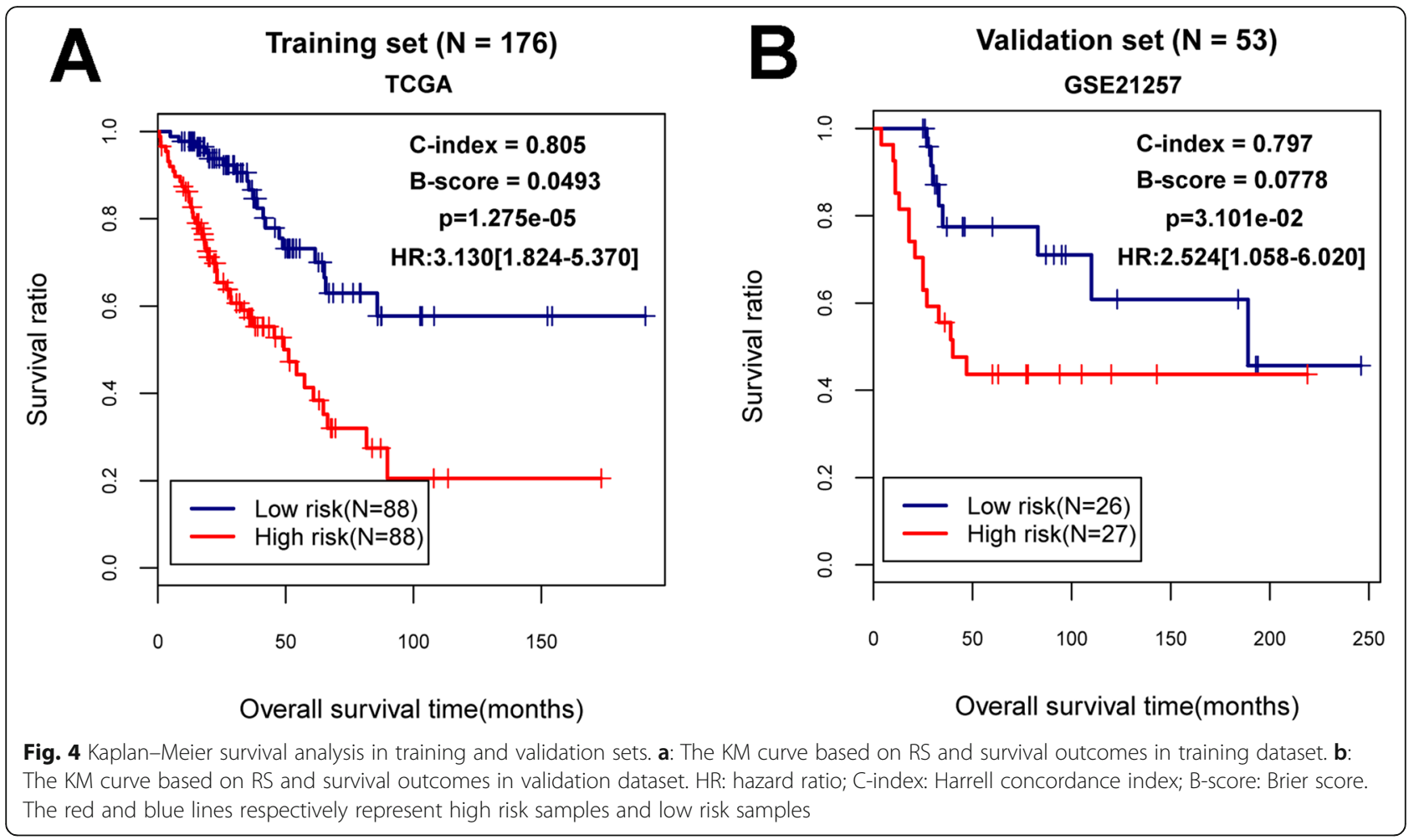

significantly up-regulated genes and 497 significantly down-regulated genes (Table S2). The volcano plot and heatmap of gene expression were showed in Fig. 6 a and b. Furthermore, the GO-BP annotation analysis and KEGG pathway analysis were undertaken for these DEGs. Our results indicated that these genes primarily played essential roles in 23 GO-BP terms including immune responses. Meanwhile, they were enriched in 8
KEGG pathways such as cytokine-cytokine receptor interaction pathway (Table 4).

\section{Discussion}

Existing evidence has demonstrated that it is crucial to identify several key gene makers related to OS survival prognosis, which provides important theoretical references for developing promising therapeutic strategies for

Table 3 The univariables and multi-variables cox regression of clinical parameters and survival outcomes of patients with osteosarcoma

\begin{tabular}{|c|c|c|c|c|c|c|c|}
\hline \multirow[t]{2}{*}{ Clinical characteristics } & \multirow[t]{2}{*}{$\operatorname{TCGA}(\boldsymbol{N}=176)$} & \multicolumn{3}{|c|}{ Uni-variables cox } & \multicolumn{3}{|c|}{ Multi-variables cox } \\
\hline & & $\mathrm{HR}$ & $95 \% \mathrm{Cl}$ & $P$ & $\mathrm{HR}$ & $95 \% \mathrm{Cl}$ & $P$ \\
\hline Age(years, mean \pm sd) & $61.01 \pm 15.23$ & 1.018 & $0.999-1.036$ & 5.19E-02 & - & - & - \\
\hline Gender(Male/Female) & $72 / 104$ & 1.102 & $0.666-1.827$ & 7.05E-01 & - & - & - \\
\hline Pathologic tumor depth(years, mean \pm sd) & $6.35 \pm 3.69$ & 1.136 & $0.951-1.227$ & 9.05E-02 & - & - & - \\
\hline Pathologic tumor length(years, mean \pm sd) & $11.76 \pm 7.25$ & 1.061 & $0.993-1.092$ & 5.91E-02 & - & - & - \\
\hline Pathologic tumor width(years, mean $\pm s d$ ) & $8.78 \pm 5.51$ & 1.091 & $0.940-1.143$ & 2.17E-01 & - & - & - \\
\hline Tumor multifocal(Yes/No/-) & $34 / 132 / 10$ & 1.685 & $0.949-2.991$ & 7.13E-02 & - & - & - \\
\hline Tumor recurrence(Yes/No/-) & $28 / 141 / 7$ & 2.692 & $1.581-4.585$ & 1.48E-04 & 1.626 & $0.914-2.893$ & $9.80 \mathrm{E}-02$ \\
\hline Tumor metastatic(Yes/No/-) & $58 / 118$ & 2.898 & $1.754-4.789$ & 1.36E-05 & 1.879 & $1.079-3.274$ & $2.58 \mathrm{E}-02$ \\
\hline Radiotherapy(Yes/No/-) & $64 / 110 / 2$ & 0.799 & $0.474-1.347$ & 3.99E-01 & - & - & - \\
\hline Tumor necrosis(No/Slight/Moderate/Severe/-) & $61 / 35 / 59 / 10 / 11$ & 1.191 & $0.925-1.530$ & 1.75E-01 & - & - & - \\
\hline RS model status(High/ Low) & $88 / 88$ & 3.13 & $1.824-5.370$ & $1.28 \mathrm{E}-05$ & 2.288 & $1.291-4.057$ & 4.60E-03 \\
\hline Dead(Death/Alive/-) & $63 / 113$ & - & - & - & - & - & - \\
\hline Overall survival time(months, mean $\pm \mathrm{sd}$ ) & $39.76 \pm 32.24$ & - & - & - & - & - & - \\
\hline
\end{tabular}




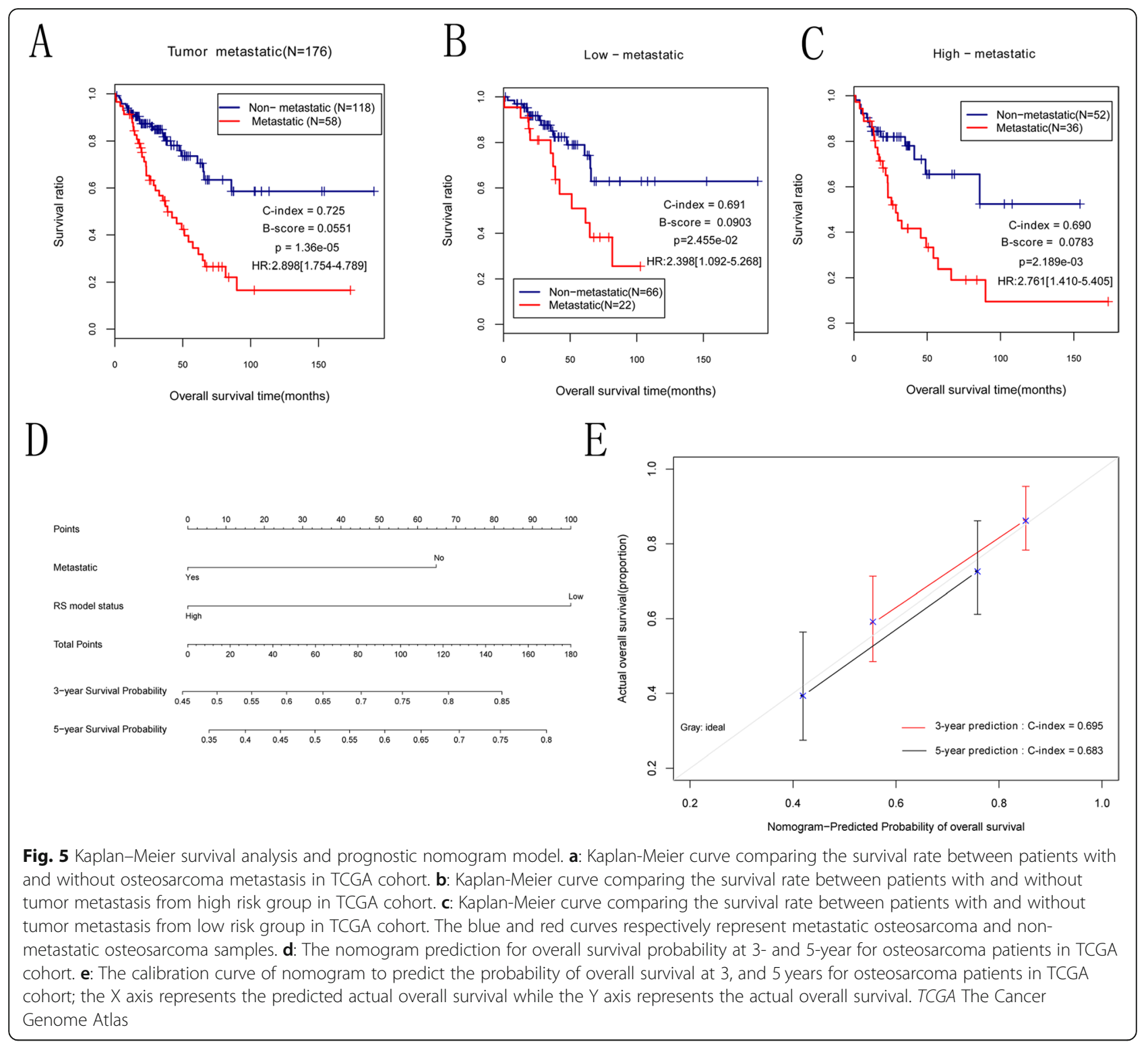

OS treatment. Herein, we established a SVM-based classifier to distinguish metastatic OS samples and nonmetastatic OS samples. Moreover, eight independent prognostic genes were identified to construct a RS model. Meanwhile, tumor metastasis and RS model status were found to serve as independent prognostic factors for OS survival. Additionally, the functional analyses of prognosis-related genes reveled that they were significantly enriched in the GO-BP term of immune responses and cytokine-cytokine receptor interaction pathway.

Tumor metastasis is a leading cause of high mortality rates of various tumors. In recent decades, the highthroughput sequencing technologies have greatly facilitated the understanding of metastasis-related genes function by decoding the genome of cancer patients
[26]. An increasing number of researchers have also concentrated on exploring the underlying pathogenesis of OS metastasis [27, 28]. Moreover, building a prediction model of OS metastasis was growingly important for prognostication and clinical decision-making. A SVM, which could effectively distinguish entities into different classes in analyzing microarray data, was frequently used in constructing sample classification model due to its high accuracy and flexibility for modeling multisource data [29]. He et al established a SVM classifier using 64 feature genes for OS and this classifier differentiated metastatic OS samples from non-metastatic OS samples in the dataset GSE21257 with a prediction accuracy of $100 \%$ [30]. Herein, we constructed a SVM classifier based on 45 prognosis-related genes to discriminate OS metastasis samples and non-metastasis samples. The 


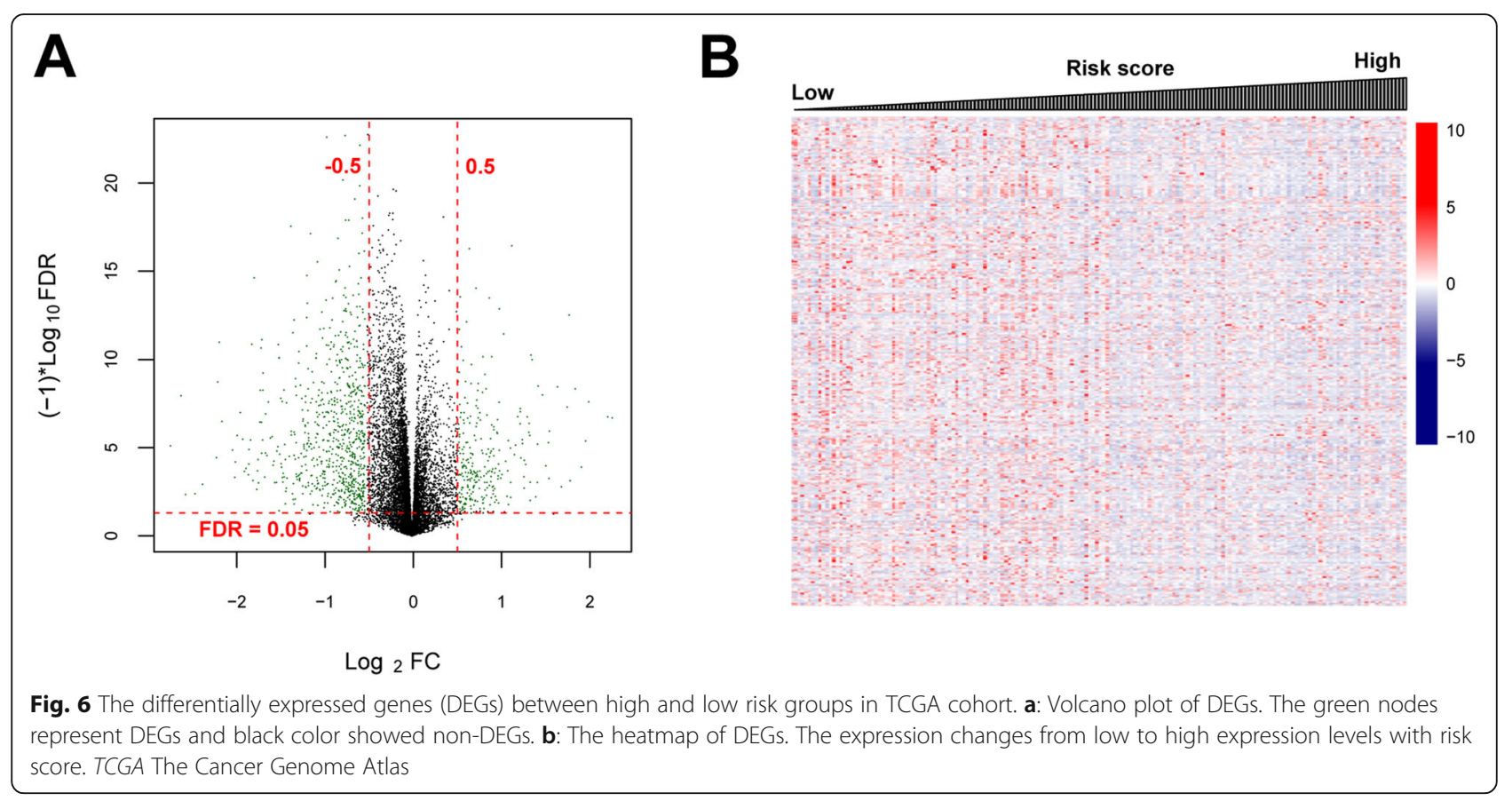

performance evaluation analysis revealed that this classifier had high precision with AUC of 0.969 , sensitivity of 0.915 and specificity of 0.884 . Moreover, these results generated in training set were also verified by a SVM classification in validation set GSE21257. The SVM classifier also exhibited a good performance with AUC of 0.907 , sensitivity of 0.857 and specificity of 0.778 . These findings implied that 45 prognostic genes might be key biomarkers to identify metastatic and non-metastatic OS patients.

The correlations between these prognosis-related genes and clinical survival were investigated by a multivariate logistic regression analysis. The results showed that there were eight independent prognostic risk genes for OS, which consisted of KCNJ15, SLC24A4, ASPA, REM1, SCARA5, LANCL3, CPA6 and TRH. A RS model was also constructed to divide OS patients into high and low-risk groups. Consequently, this eight-gene signature exhibited a good performance to differentiate metastatic OS patients from non-metastatic OS patients. KCNJ15 (also known as KIR4.2), belongs to a member of the KIR4 subfamily and encodes the potassium channel. Liu et al recently reported the silencing of $K C N J 15$ played key roles in tumor malignance and was related to unfavourable prognosis renal carcinoma [31]. However, whether KCNJ15 directly involves in OS progression has not been clarified. Notably, multiple investigations have demonstrated that some potassium ion channels such as hSlo potassium channel in OS cells were implicated with the carcinogenesis [32, 33]. Therefore, the potential roles of $K C N J 15$ in pathogenesis of OS need to be investigated in future. SLC24A4/NCKX4 is located at on chromosome region $14 \mathrm{q} 32$ and a member of potassiumdependent sodium-calcium exchanger gene family [34]. No reports are concerned about the relationship of this gene and OS development. REM1 encodes a GTPase and participates regulating the activity of voltagedependent $\mathrm{Ca}^{2+}$ channels. Numerous studies have suggested that SCARA5, a member of the scavenger receptor family, is involved in the molecular mechanisms of various cancers such as hepatocellular carcinoma [35]. You et al previously found that SCARA5 served as a key biomarker for the development and metastasis of breast cancer [36]. An early research reported that CPA6 was remarkably up-regulated in early stage samples with oral squamous cell carcinoma (OSCC) compared with those in late stage, suggesting that this gene might have crucial diagnostic values for OSCC [37]. Another study emphasized that the methylation of TRH could classify OSCC and oropharyngeal SCC patients from healthy individuals with a high accuracy [38]. Unfortunately, the association of eight independent prognostic genes and OS has not been unraveled until now. Further investigations are essential to understand the underlying role of these genes for the diagnosis and prediction of OS.

Additionally, the DEGs between high and low risk groups in training dataset were further extracted to understand the influence of this eight-gene signature on OS prognosis prediction. There were 614 significantly DEGs (117 up-regulated and 497 down-regulated genes). The GO-BP analysis indicated that these genes were mainly responsible for immune responses. Mori et al 
Table 4 The functional analyses of survival-related genes

\begin{tabular}{|c|c|c|c|c|}
\hline Category & Term & Count & $\boldsymbol{P}$ value & FDR \\
\hline \multirow[t]{23}{*}{ GO-BP } & GO:0002250 adaptive immune response & 28 & $6.430 \mathrm{E}-13$ & 1.560E-09 \\
\hline & GO:0050776 regulation of immune response & 28 & $5.700 \mathrm{E}-11$ & $6.900 \mathrm{E}-08$ \\
\hline & GO:0070098 chemokine-mediated signaling pathway & 18 & $1.450 \mathrm{E}-10$ & 1.170E-07 \\
\hline & GO:0006955 immune response & 41 & 3.820E-09 & 1.850E-06 \\
\hline & GO:0042110 T cell activation & 14 & $3.170 \mathrm{E}-09$ & $1.920 \mathrm{E}-06$ \\
\hline & GO:0006935 chemotaxis & 19 & $1.430 \mathrm{E}-07$ & $5.750 \mathrm{E}-05$ \\
\hline & GO:0006954 inflammatory response & 35 & $2.300 \mathrm{E}-07$ & 7.950E-05 \\
\hline & GO:0006968 cellular defense response & 13 & $9.040 \mathrm{E}-07$ & $2.730 \mathrm{E}-04$ \\
\hline & GO:0007267 cell-cell signaling & 26 & $1.760 \mathrm{E}-06$ & 4.740E-04 \\
\hline & GO:0007166 cell surface receptor signaling pathway & 27 & 2.180E-06 & $5.280 \mathrm{E}-04$ \\
\hline & GO:0071346 cellular response to interferon-gamma & 11 & $1.840 \mathrm{E}-05$ & 4.037E-03 \\
\hline & GO:0042102 positive regulation of T cell proliferation & 11 & $2.930 \mathrm{E}-05$ & 5.894E-03 \\
\hline & GO:0007204 positive regulation of cytosolic calcium ion concentration & 16 & 4.730E-05 & 8.769E-03 \\
\hline & GO:0035589 G-protein coupled purinergic nucleotide receptor signaling pathway & 6 & $6.700 \mathrm{E}-05$ & $1.152 \mathrm{E}-02$ \\
\hline & GO:0043547 positive regulation of GTPase activity & 38 & 9.630E-05 & $1.446 \mathrm{E}-02$ \\
\hline & GO:0070374 positive regulation of ERK1 and ERK2 cascade & 18 & $9.260 \mathrm{E}-05$ & 1.484E-02 \\
\hline & GO:0007155 cell adhesion & 32 & $2.060 \mathrm{E}-04$ & 2.894E-02 \\
\hline & GO:0007165 signal transduction & 63 & $2.210 \mathrm{E}-04$ & $2.926 \mathrm{E}-02$ \\
\hline & GO:0048247 lymphocyte chemotaxis & 7 & $2.880 \mathrm{E}-04$ & 3.606E-02 \\
\hline & GO:0042472 inner ear morphogenesis & 9 & $3.260 \mathrm{E}-04$ & 3.873E-02 \\
\hline & GO:0050850 positive regulation of calcium-mediated signaling & 6 & $4.390 \mathrm{E}-04$ & 4.712E-02 \\
\hline & GO:0006508 proteolysis & 33 & 4.240E-04 & 4.774E-02 \\
\hline & GO:0002548 monocyte chemotaxis & 8 & 4.660E-04 & 4.781E-02 \\
\hline \multirow[t]{8}{*}{ KEGG Pathway } & hsa04060:Cytokine-cytokine receptor interaction & 38 & 4.250E-12 & 9.690E-10 \\
\hline & hsa04080:Neuroactive ligand-receptor interaction & 34 & 4.100E-08 & 4.680E-06 \\
\hline & hsa04640:Hematopoietic cell lineage & 17 & $4.960 \mathrm{E}-07$ & 3.770E-05 \\
\hline & hsa04514:Cell adhesion molecules (CAMs) & 19 & $2.440 \mathrm{E}-05$ & 1.389E-03 \\
\hline & hsa04062:Chemokine signaling pathway & 21 & $9.560 \mathrm{E}-05$ & 4.350E-03 \\
\hline & hsa04660:T cell receptor signaling pathway & 13 & $9.200 \mathrm{E}-04$ & $2.590 \mathrm{E}-02$ \\
\hline & hsa05033:Nicotine addiction & 8 & $1.213 \mathrm{E}-03$ & $3.028 \mathrm{E}-02$ \\
\hline & hsa04650:Natural killer cell mediated cytotoxicity & 14 & $1.739 \mathrm{E}-03$ & $3.543 \mathrm{E}-02$ \\
\hline
\end{tabular}

KEGG Kyoto Encyclopedia of Genes and Genomes, FDR false discovery rate, GO-BP Gene Ontology-Biology Process

argued that up-regulation of the immune response was a critical characteristic in patients with tumors and several immunotherapies were potent approaches for those patients undergoing OS metastasis [39]. Moreover, a wealth of evidence has suggested that the involvement of immunotherapies could regulate the tumor microenvironment and re-activate prolonged immune responses $[40,41]$. The results of KEGG enrichment analysis showed that these genes were predominately associated with cytokine-cytokine receptor interaction pathway. Similarly, Chen et al performed a bioinformatics analysis based on a circRNA microarray dataset and three gene expression profiles of OS cell lines. They found that those down-regulated DEGs from three gene profiles mainly played prominent roles in cytokine-cytokine receptor interaction pathway [42]. These findings revealed that the initiation of immune responses and cytokinecytokine receptor interaction possibly contributed to the OS progression.

In the current study, we also found tumor metastasis and RS model status acted as independent prognostic factors for OS survival by the cox regression model analysis. Then, these two survival-related factors as variables were incorporated into the nomogram and the results indicated that RS model status showed the biggest influence on OS survival prognosis. The nomogram is a powerful risk assessment tool for a wide variety of diseases including OS, which provides important guidance 
for clinical outcomes prediction, therapy selection and follow-up care [43]. We noted that overall survival rates the 3- and 5-year for OS patients were similar to the actual observation for OS patients, implying that tumor metastasis and RS model status were vital clinical characteristics for survival prediction of OS patients.

Although an eight-gene panel and two independent prognostic factors have been identified to be associated with OS prognosis, the detailed pathologic mechanisms have not been elucidated. For example, whether these gene signatures are involved in several molecular pathways such as cytokine-cytokine receptor interaction pathway still needs to be illuminated. Moreover, a further accurate classification with a large sample size and clinical information is necessary to distinguish OS metastasis and non-metastasis patients. In addition, the external validation is not carried out to check the reliability of our nomogram. Meanwhile, the performance evaluation of nomogram established here also requires to be performed. Finally, the corresponding experimental research is also needed to verify the biological functions of key gene.

In conclusion, we constructed a SVM-based classifier to separate metastatic and non-metastatic patients. Moreover, the eight-gene signature and two independent prognostic factors (tumor metastasis and RS model status) were closely related to OS survival. These findings greatly improved the understandings of OS metastasis and prognosis. However, relevant validation studies and optimization of prognostic model for OS will be considered in future.

\section{Conclusion}

An eight-gene predictive model and nomogram were developed to predict OS prognosis.

\section{Supplementary information}

Supplementary information accompanies this paper at https://doi.org/10. 1186/s12885-020-06741-4.

Additional file 1: Table S1. The list of differentially expressed genes between metastatic and non-metastatic osteosarcoma patients in training dataset was displayed. Table S2. The list of differentially expressed genes between high and low risk groups in training dataset.

\footnotetext{
Abbreviations

OS: Osteosarcoma GEO Gene expression omnibus GO Gene Ontology; TCGA: The Cancer Genome Atlas; DEGs: Differentially expressed genes; SVM: Support vector machine; DEmiRNA: Differentially expressed miRNAs; SVM-RFE: SVM recursive feature elimination; RS: Risk score; FDR: False discovery rate; FC: Fold change; OPGS: Optimal prognostic gene set; pROC: Partial receiver operating characteristic; AUC: Area under curve; PPV: Positive predictive value; NPV: Negative predictive value; RS: Risk score; KM: Kaplan-Meier; BP: Biological process; KEGG: Kyoto Encyclopedia of Genes and Genomes; HR: Hazard Ratio; Cl: Confidence intervals; OSCC: Oral squamous cell carcinoma
}

\section{Acknowledgements}

none.

\section{Authors' contributions}

GZW and MLZ participated in the design of this study, and they both performed the statistical analysis. GZW carried out the study and collected important background information. MLZ drafted the manuscript. All authors read and approved the final manuscript.

\section{Funding}

none.

Availability of data and materials

The raw data were collected and analyzed by the Authors, and are not ready to share their data because the data have not been published.

Ethics approval and consent to participate

not applicable

Consent for publication

Not applicable.

\section{Competing interests}

The authors declare that they have no competing interests.

\section{Author details}

'Departments of Hand Surgery, The Third Hospital of Jilin University, Changchun, Jilin Province, China. ${ }^{2}$ Departments of Orthopedics, The Third Hospital of Jilin University, Changchun, Jilin Province, China.

Received: 18 November 2019 Accepted: 12 March 2020

Published online: 24 May 2020

\section{References}

1. Ritter J, Bielack SS.Osteosarcoma. Ann Oncol 2010;217:320-5.

2. Bielack S, Carrle D, Casali PG, Group EGW. Osteosarcoma: ESMO clinical recommendations for diagnosis, treatment and follow-up. Ann Oncol Suppl. 2009:4:137-9.

3. Wakamatsu T, Kakunaga S, Takenaka S, Outani H, Hamada K, Imura Y, Hori Y, Naka N, Kudawara I, Yoshikawa H, Ueda T. Prognostic implication of adjuvant/neoadjuvant chemotherapy consisting of doxorubicin and ifosfamide in patients with extraskeletal osteosarcoma. Int J Clin Oncol oi. 2019. https://doi.org/10.1007/s10147-019-01475-1

4. Pei $Y$, Yao Q, Li Y, Zhang $X$, Xie B. microRNA-211 regulates cell proliferation, apoptosis and migration/invasion in human osteosarcoma via targeting EZRIN. Cell Mol Biol Lett. 2019;24:48.

5. Ferrari S, Smeland S, Mercuri M, Bertoni F, Longhi A, Ruggieri P, Alvegard TA, Picci P, Capanna R, Bernini G, Müller C, Tienghi A, Wiebe T, Comandone A, Böhling T, Del Prever AB, Brosjö O, Bacci G, Saeter G, Italian, Scandinavian Sarcoma G. Neoadjuvant chemotherapy with high-dose Ifosfamide, highdose methotrexate, cisplatin, and doxorubicin for patients with localized osteosarcoma of the extremity: a joint study by the Italian and Scandinavian Sarcoma Groups. J Clin Oncol. 2005;23(34):8845-52.

6. Siegel RL, Miller KD, Jemal A. Cancer statistics. CA Cancer J Clin. 2019;69(1): 7-34.

7. Wang X, Yu Y, He Y, Cai Q, Gao S, Yao W, Liu Z, Tian Z, Han Q, Wang W, Sun R, Luo Y, Li C. Upregulation of ALDH1B1 promotes tumor progression in osteosarcoma. Oncotarget. 2017:9(2):2502-14.

8. Shi JH, Hao YJ. DDX10 overexpression predicts worse prognosis in osteosarcoma and its deletion prohibits cell activities modulated by MAPK pathway. Biochem Biophys Res Commun. 2019;510(4):525-9.

9. He Y, Ma J, Wang A, Wang W, Luo S, Liu Y, Ye X. A support vector machine and a random forest classifier indicates a 15-miRNA set related to osteosarcoma recurrence. Onco Targets Ther. 2018;11:253-69.

10. Liu F, Xing L, Zhang X, Zhang X. A four-Pseudogene classifier identified by machine learning serves as a novel prognostic marker for survival of osteosarcoma. Gene (Basel). 2019;10(6):414.

11. Buddingh EP, Kuijjer ML, Duim RAJ, Bürger $H$, Agelopoulos $K$, Myklebost $O$, Serra M, Mertens F, Hogendoorn PCW, Lankester AC, Cleton-Jansen A-M. Tumor-infiltrating macrophages are associated with metastasis suppression in high-grade osteosarcoma: a rationale for treatment with macrophage activating agents. Clin Cancer Res. 2011;17(8):2110-9. 
12. Edgar R, Domrachev M, Lash AE.Gene expression omnibus: NCBI gene expression and hybridization array data repository. Nucleic Acids Res. 2002 30(1):207-10.

13. Ritchie ME, Phipson B, Wu D, Hu Y, Law CW, Shi W, Smyth GK. Limma powers differential expression analyses for RNA-sequencing and microarray studies. Nucleic Acids Res. 2015:43(7):e47.

14. Wang L, Cao C, Ma Q, Zeng Q, Wang H, Cheng Z, Zhu G, Qi J, Ma H, Nian $H$, Wang Y. RNA-seq analyses of multiple meristems of soybean: novel and alternative transcripts, evolutionary and functional implications. BMC Plant Biol. 2014;14:169.

15. Wang P, Wang Y, Hang B, Zou X, Mao JH . A novel gene expression-based prognostic scoring system to predict survival in gastric cancer. Oncotarget. 2016;7(34):55343-51.

16. Wang $Q$, Liu X. Screening of feature genes in distinguishing different types of breast cancer using support vector machine. Onco Targets Ther. 2015;8: 2311-7

17. Deist TM, Dankers FJWM, Valdes G, Wijsman R, Hsu IC, Oberije C, Lustberg T, van Soest J, Hoebers F, Jochems A, El Naqa I, Wee L, Morin O, Raleigh DR, Bots W, Kaanders JH, Belderbos J, Kwint M, Solberg T, Monshouwer R, Bussink J, Dekker A, Lambin P. Machine learning algorithms for outcome prediction in (chemo)radiotherapy: an empirical comparison of classifiers. Med Phys. 2018;45(7):3449-59.

18. Lu X, Yang Y, Wu F, Gao M, Xu Y, Zhang Y, Yao Y, Du X, Li C, Wu L, Zhong $X$, Zhou $Y$, Fan $N$, Zheng $Y$, Xiong D, Peng H, Escudero J, Huang B, Li X, Ning $Y$, Wu K.Discriminative analysis of schizophrenia using support vector machine and recursive feature elimination on structural MRI images. Medicine (Baltimore). 2016;95(30):e3973.

19. Robin X, Turck N, Hainard A, Tiberti N, Lisacek F, Sanchez J-C, Müller M. PROC: an open-source package for R and $\mathrm{S}+$ to analyze and compare ROC curves. BMC Bioinformatics. 2011;12:77.

20. Mayr A, Schmid M. Boosting the concordance index for survival data--a unified framework to derive and evaluate biomarker combinations. PLoS One. 2014;9(1):e84483.

21. Zhang $X, L i$ Y, Akinyemiju T, Ojesina Al, Buckhaults $P$, Liu N, Xu B, Yi N. Pathway-structured predictive model for Cancer survival prediction: a twostage approach. Genetics. 2017;205(1):89-100.

22. Anderson WI, Schlafer DH, Vesely KR. Thyroid follicular carcinoma with pulmonary metastases in a beaver (Castor canadensis). J Wildl Dis. 1989; 25(4):599-600.

23. Schröder MS, Culhane AC, Quackenbush J, Haibe-Kains B. Survcomp: an R/ bioconductor package for performance assessment and comparison of survival models. Bioinformatics. 2011;27(22):3206-8.

24. Eng KH, Schiller E, Morrell K. On representing the prognostic value of continuous gene expression biomarkers with the restricted mean survival curve. Oncotarget. 2015;6(34):36308-18

25. Eisen MB, Spellman PT, Brown PO, Botstein D.Cluster analysis and display of genome-wide expression patterns. Proc Natl Acad Sci U S A. 1998;95(25): 14863-8.

26. Yuan L, Guo F, Wang L, Zou Q.Prediction of tumor metastasis from sequencing data in the era of genome sequencing. Brief Funct Genomics. 2019. https://doi.org/10.1093/bfgp/elz010 .

27. Wang S, Zhong L, Li Y, Xiao D, Zhang R, Liao D, Lv D, Wang X, Wang J, Xie $X$, Chen J, Wu Y, Kang T. Up-regulation of PCOLCE by TWIST1 promotes metastasis in osteosarcoma. Theranostics. 2019;9(15):4342-53.

28. Scott MC, Temiz NA, Sarver AE, LaRue RS, Rathe SK, Varshney J, Wolf NK, Moriarity BS, O'Brien TD, Spector LG, Largaespada DA, Modiano JF, Subramanian S, Sarver AL. Comparative Transcriptome analysis quantifies immune cell transcript levels, metastatic progression, and survival in osteosarcoma. Cancer Res. 2018:78(2):326-37.

29. Furey TS, Cristianini N, Duffy N, Bednarski DW, Schummer M, Haussler D. Support vector machine classification and validation of cancer tissue samples using microarray expression data. Bioinformatics. 2000;16(10):90614.

30. He Y, Ma J, Ye X. A support vector machine classifier for the prediction of osteosarcoma metastasis with high accuracy. Int J Mol Med. 2017:40(5): 1357-64.

31. Liu Y, Wang H, Ni B, Zhang J, Li S, Huang Y, Cai Y, Mei H, Li Z.Loss of KCNJ15 expression promotes malignant phenotypes and correlates with poor prognosis in renal carcinoma. Cancer Manag Res. 2019;11:1211-20.

32. Cambien B, Rezzonico R, Vitale $S$, Rouzaire-Dubois B, Dubois J-M, Barthel $R$, Soilihi BK, Mograbi B, Schmid-Alliana A, Schmid-Antomarchi H. Silencing of
hSlo potassium channels in human osteosarcoma cells promotes tumorigenesis. Int J Cancer. 2008;123(2):365-71.

33. Rezzonico R, Schmid-Alliana A, Romey G, Bourget-Ponzio I, Breuil V, Breittmayer $\mathrm{V}$, Tartare-Deckert S, Rossi B, Schmid-Antomarchi H. Prostaglandin E2 induces interaction between hSlo potassium channel and Syk tyrosine kinase in osteosarcoma cells. J Bone Miner Res. 2002;17(5):86978.

34. Li XF, Kraev AS, Lytton J. Molecular cloning of a fourth member of the potassium-dependent sodium-calcium exchanger gene family, NCKX4. J Biol Chem. 2002;277(50):48410-7.

35. Liu H, Hu J, Wei R, Zhou L, Pan H, Zhu H, Huang M, Luo J, Xu W.SPAG5 promotes hepatocellular carcinoma progression by downregulating SCARA5 through modifying $\beta$-catenin degradation. J Exp Clin Cancer Res. 2018;37(1): 229.

36. You K, Su F, Liu L, LV X, Zhang J, Zhang Y, Liu B. SCARA5 plays a critical role in the progression and metastasis of breast cancer by inactivating the ERK1/ 2, STAT3, and AKT signaling pathways. Mol Cell Biochem. 2017;35(1-2):4758.

37. Fialka F, Gruber RM, Hitt R, Opitz L, Brunner E, Schliephake H, Kramer FJ. CPA6, FMO2, LGI1, SIAT1 and TNC are differentially expressed in early- and late-stage Oral squamous cell carcinoma--a pilot study. Oral Oncol. 2008; 44(10):941-8.

38. Puttipanyalears C, Arayataweegool A, Chalertpet K, Rattanachayoto P, Mahattanasakul P, Tangjaturonsasme N, Kerekhanjanarong V, Mutirangura A, Kitkumthorn NJBC.TRH site-specific methylation in oral and oropharyngeal squamous cell carcinoma. BMC Cancer. 2018;18(1):786.

39. Mori K, Redini F, Gouin F, Cherrier B, Heymann D.Osteosarcoma: current status of immunotherapy and future trends (review). Oncol Rep. 2006;15(3): 693-700.

40. Heymann MF, Brown HK, Heymann D.Drugs in early clinical development for the treatment of osteosarcoma. Expert Opin Investig Drugs. 2016;25(11): 1265-80.

41. Heymann MFO, Lézot F, Heymann DJCl. The contribution of immune infiltrates and the local microenvironment in the pathogenesis of osteosarcoma. Cell Immunol pii. 2017;S0008-8749(17):30189-2.

42. Chen G, Wang Q, Yang Q, Li Z, Du Z, Ren M, Zhao H, Song Y, Zhang G. Circular RNAs hsa_circ_0032462, hsa_circ_0028173, hsa_circ_0005909 are predicted to promote CADM1 expression by functioning as miRNAs sponge in human osteosarcoma. PLoS One. 2018;13(8):e0202896.

43. Zhang J, Yang J, Wang HQ, Pan Z, Yan X, Hu C, Li Y, Lyu J. Development and validation of a nomogram for osteosarcoma-specific survival. Medicine (Baltimore). 2019:98(23):e15988.

\section{Publisher's Note}

Springer Nature remains neutral with regard to jurisdictional claims in published maps and institutional affiliations.
Ready to submit your research? Choose BMC and benefit from:
- fast, convenient online submission
- thorough peer review by experienced researchers in your field
- rapid publication on acceptance
- support for research data, including large and complex data types
- gold Open Access which fosters wider collaboration and increased citations
- maximum visibility for your research: over $100 \mathrm{M}$ website views per year
At BMC, research is always in progress.
Learn more biomedcentral.com/submissions 